\title{
Demonstration of Parallel Scanning Probe Microscope for High Throughput Metrology and Inspection
}

\author{
Hamed Sadeghian ${ }^{1,2}$, Bert Dekker ${ }^{1}$, Rodolf Herfst ${ }^{1}$, Jasper Winters ${ }^{1}$, Alexander Eigenraam ${ }^{1}$, Ramon \\ Rijnbeek $^{1}$, Nicole Nulkes ${ }^{1}$ \\ ${ }^{1}$ Department of Optomechatronics, Netherlands Organization for Applied Scientific Research, TNO \\ Delft, The Netherlands \\ ${ }^{2}$ Department of Precision and Microsystems Engineering, Delft University of Technology, Delft, \\ The Netherlands
}

\begin{abstract}
With the device dimensions moving towards the $1 \mathrm{X}$ node and below, the semiconductor industry is rapidly approaching the point where existing metrology, inspection and review tools face huge challenges in terms of resolution, the ability to resolve 3D and the throughput. Due to the advantages of sub-nanometer resolution and the ability of true 3D scanning, scanning probe microscope (SPM) and specifically atomic force microscope (AFM) are considered as alternative technologies for CD-metrology, defect inspection and review of $1 \mathrm{X}$ node and below.

In order to meet the increasing demand for resolution and throughput of CD-metrology, defect inspection and review, TNO has previously introduced the parallel SPM concept, consisting of parallel operation of many miniaturized SPMs on a 300 and $450 \mathrm{~mm}$ wafer. In this paper we will present the proof of principle of the parallelization for metrology and inspection. To give an indication of the system's specifications, the throughput of scanning is 4500 sites per hour, each within an area of $1 \mu \mathrm{m}^{2}$ and $1024 \times 1024$ pixels.
\end{abstract}

Keywords: parallel atomic force microscope, scanning probe microscope, wafer, mask, CD-metrology, Defect review, process control

\section{INTRODUCTION}

With the shrinking of the device size towards $10 \mathrm{~nm}$ and increasing device complexity, the existing metrology solutions are pushed to their limits of performance. The challenges of metrology and inspection is two folded:

1) The size of the features and killer defects becomes so small that the current methods are not anymore capable of reliable measurement at the required throughput in a high volume manufacturing (HVM) environment (ITRS2013).

2) The architecture of devices is becoming more complex, i.e. 3D which poses another challenge for metrology. Resolving of the 3D shape is necessary in order to have a meaningful metrology. Taking into account the required throughput, there are currently no methods capable of resolving 3D with sufficient resolution at the required throughput with no damage to the features.

The recent developments in the field of scanning probe microscopy (SPM) have shown that this method can be considered as an option for metrology and inspection. This comes from the fact that SPM is capable of resolving 3D structures at very high spatial resolution. Moreover, since SPM is capable of measuring several physical characteristics (i.e. elasticity, adhesion) it can be used for defect classifications too. The main bottleneck of this method was the very low throughput of measurement as well as possible damage to the substrate if throughput is met.

There have been several attempts to increase the speed of single SPM further, but so far it is not yet at a level to be able to compete with other metrology and inspection systems in throughput [1,2]. Recently TNO has developed a concept for high throughput SPM measurement [3]. The increase in throughput was a results of two improvements:

1) Sufficient miniaturization of the SPM to operate many of them in parallel.

Metrology, Inspection, and Process Control for Microlithography XXIX, edited by Jason P. Cain,

Martha I. Sanchez, Proc. of SPIE Vol. 9424, 942400 - (c) 2015 SPIE

CCC code: $0277-786 X / 15 / \$ 18 \cdot$ doi: $10.1117 / 12.2085495$

Proc. of SPIE Vol. 9424 942400-1 
2) A speed increase of the mini SPM by pushing the bandwidth of the SPM's sub-modules, i.e mechanical stages (x,y and z), optical read-out, controller bandwidth, approach speed and the speed of positioning. We have experimentally demonstrated the feasibility of such a high speed mini SPM and a mini positioning unit capable of positioning each scanhead very fast and accurate.

This paper presents the developments towards experimental demonstration of parallel SPM system, where a number mini SPMs scan several sites on a wafer. This proves the feasibility of such a parallel SPM system and satisfies the requirement of HVM metrology and review in terms of resolution and throughput.

\section{PARALLEL SPM ARCHITECTURE}

The core of the architecture is based on a miniature SPM and a miniature positioning unit to position the SPM at the scanned location. The goal is to parallelize many of such mini SPM and positioning unit to be able to scan many sites in parallel. Figure 1 shows the concept of such parallel system $[3,7]$.

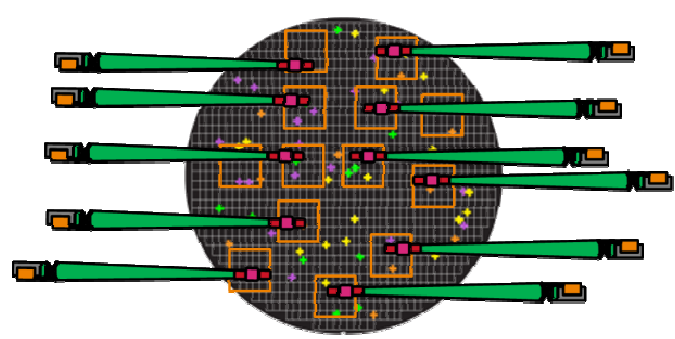

(a)

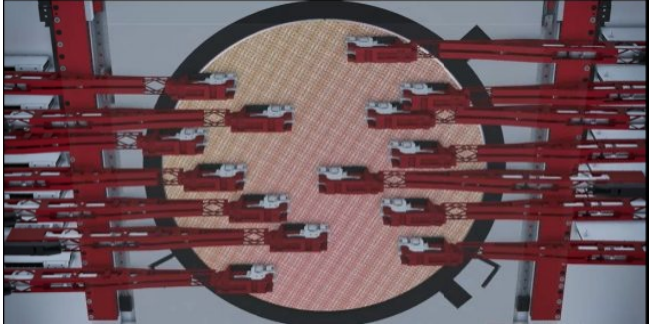

(b)

Figure 1(a) Concept of parallel SPM to image several locations on a wafer or mask. (b) detail with wafer stage in the middle, multiple positioning arms on two sides of the wafer stage, each capable of moving a SPM scanhead on to the $450 \mathrm{~mm}$ wafer. The figure is taken from [3].

Last year we have shown the proof of principle and the experimental results of one single unit that consists of an ultrafast miniature AFM head (scanhead) and an accurate, fast positioning unit to position the scanhead with respect to a wafer [4]. The system was capable of scanning areas on a wafer as large as $100 \times 100 \mu \mathrm{m}^{2}$ in several tenths of seconds. The scanhead showed a measurement bandwidth of $50 \mathrm{KHz}$, and a resolution as low as $0.1 \mathrm{~nm}$. The closed loop positioning unit results in an positioning accuracy better than $100 \mathrm{~nm}$.

Figure 2 shows the first demonstrator version of a single miniature AFM and its positioning unit.

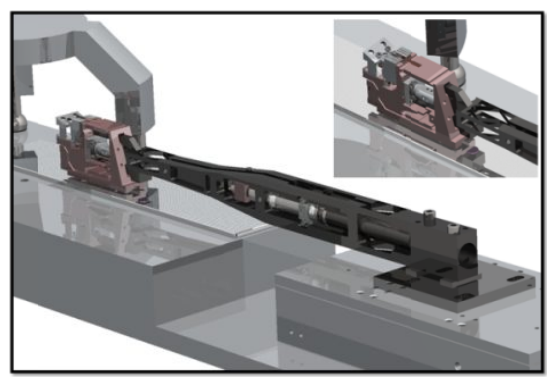

(a)

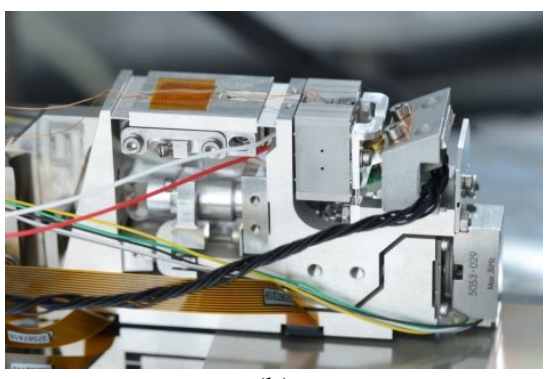

(b) 


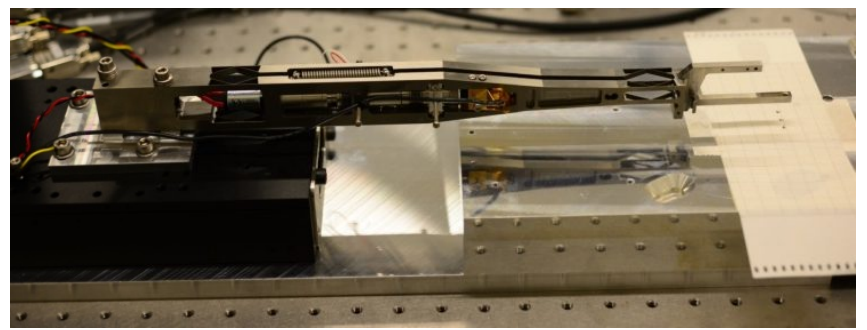

(c)

Figure 2: Top left: final design of one scanhead and one arm. Top, right: Proof of concept of miniature AFM. Bottom: proof of principle of the positioning unit.

Obviously due to the large number of wires, this version was not suitable for the final parallelization and therefore several improvements have been made in the second version of the scanhead and arm.

In the next step we have designed and realized the final parallel AFM system to experimentally show the feasibility of parallel scanning on both wafer and mask. The system consists of 4 parallel miniature scanheads and positioning units that simultaneously scan a wafer or a mask. The system can be scaled up easily to more than 40 parallel units. The user can insert several locations on a mask and wafer to be scanned.

To successfully integrate multiple arms and scanheads into a full system, each arm and scanhead combination needs to be contained within a narrowly defined area. In our first system this was not yet the case, as all the electrical components were connected with individual wires and cables. To streamline this we have integrated the required electrical connections into flex-rigid PCBs that are glued to the arm. Because of this, the electrical connections have become longer, leading to somewhat higher resistances and an increased risk of cross-talk and higher noise that could lower the performance of the scanhead. In the section "Results and Discussion" we will show that this is not the case; high quality scans were made with the new system including the flex-rigid PCBs. Figure 3 shows the final design of the parallel SPM system.

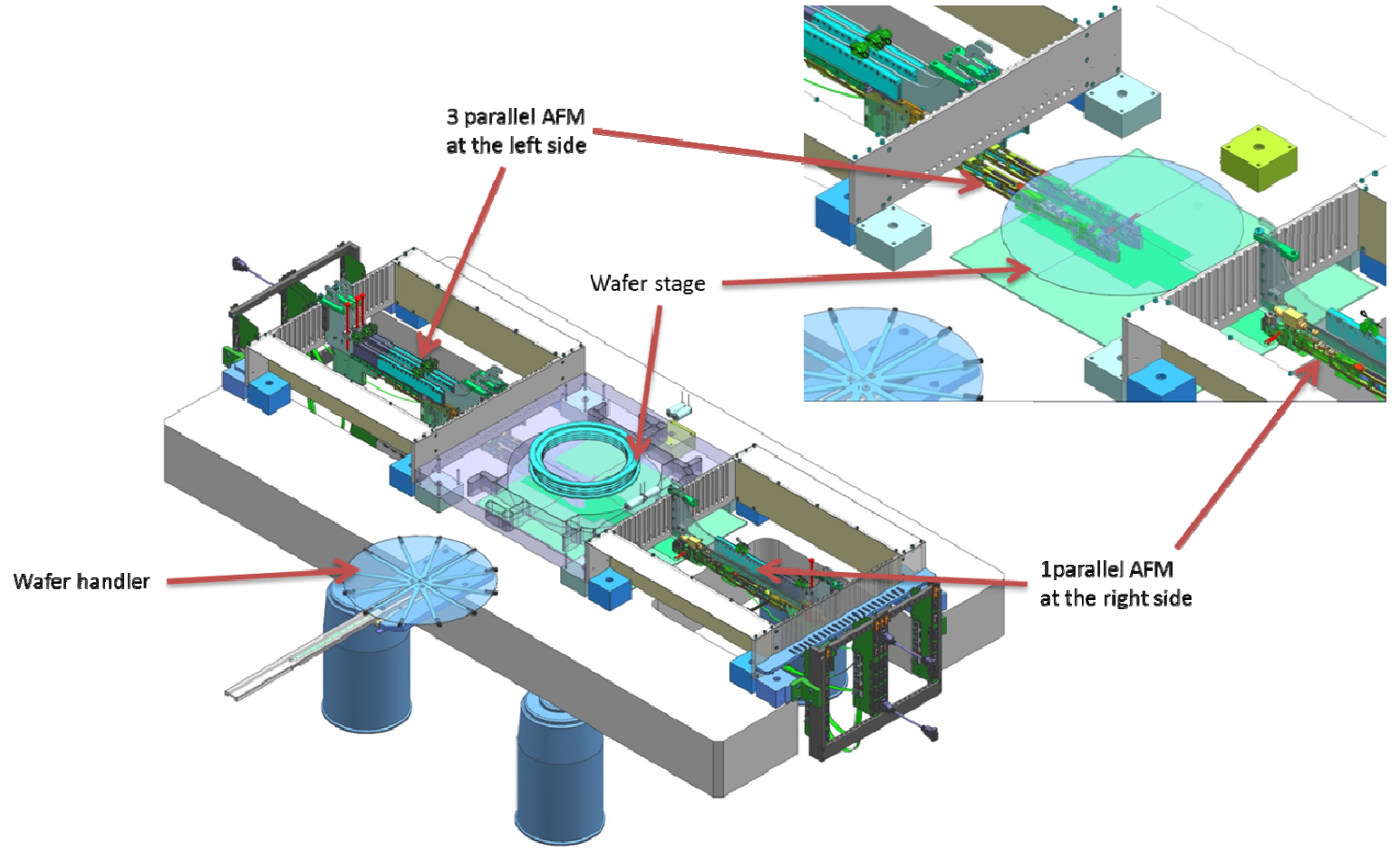

Figure 3:CAD design of the final 4 parallel AFM system for wafer and mask. 
To summarizze, in our second version of the parallel SPM we have realized:

- The integration of all the required electrical connections into flex-rigid PCBs that are glued to the arm.

- A wafer stage capable of moving 100,300 and $450 \mathrm{~mm}$ wafers with a stroke range of $50 \mu \mathrm{m}$.

- Integration of miniature linear motors for actuation of the arm in the x-direction.

- Sensors for monitoring the correct disengaging of the scanhead from the arm.

- A cassette system for placing and exchanging the arm and scanhead combinations.

- Parallelization of the control system.

- Various improvements with respect to aligning of the system and mechanical isolation of the scanhead.

\section{METROLOGY AND MEASURMENT LOOP}

The position of the cantilever tip with respect to the wafer and targeted area must be known before and during AFM measurements. This metrology loop is illustrated in Figure 4.

Capacitive sensors in the wafer stage provide real-time feedback of the chuck position. A large grid plate is placed directly on the stone and provides the location reference for the scanheads. The scanheads all incorporate positioning sensors that measure its relative position to the reference lines of the grid plate in $\mathrm{X}$ and $\mathrm{Y}$ direction and also in rotation around the Z-axis.

Each scanhead is calibrated to determine the position of its cantilever tip w.r.t. its base. This is also required after automated tip exchange.

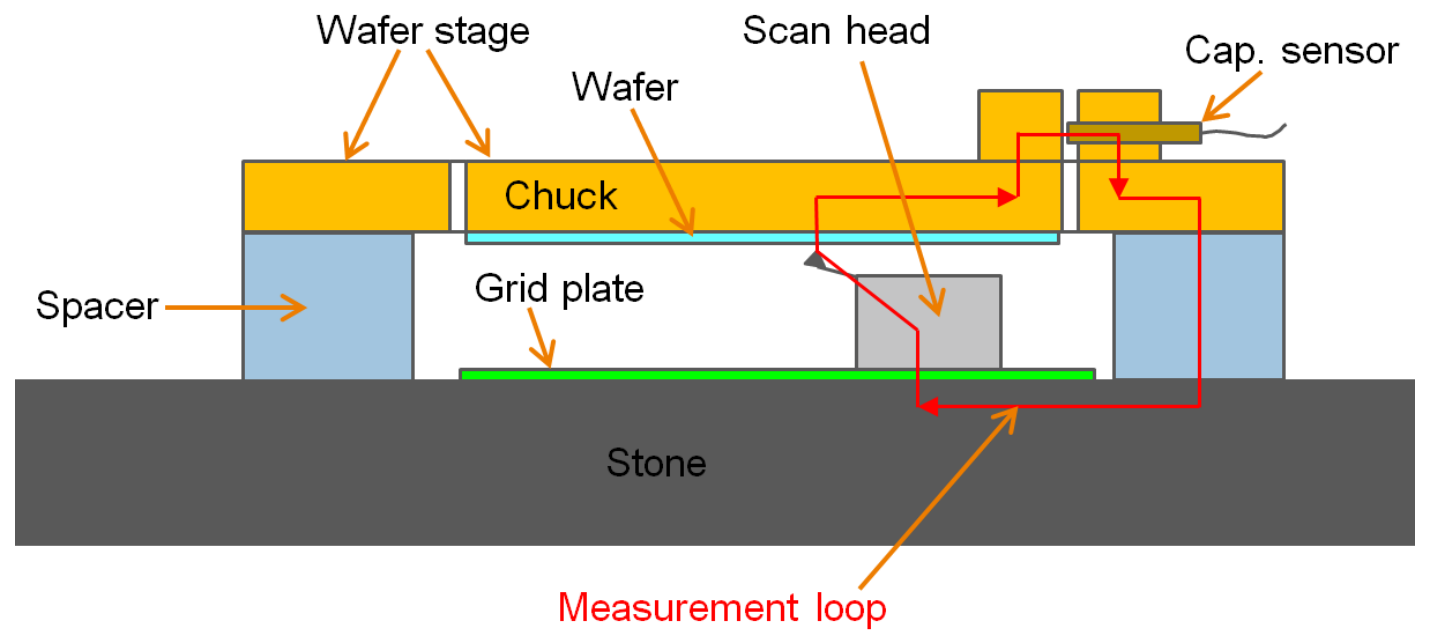

Figure 4: Metrology loop in parallel SPM.

Besides the scanhead, the other parts in the measurement loop are the wafer, wafer stage, capacitive sensor mounts, stone and grid plate. Special effort has been put in making the stiffness of the parts in this loop high enough to not negatively influence the AFM measurements. The accelerations of the chuck and the impact on the chuck-wafer interface have been investigated to check for slipping at this interface and we found it will have no impact on the measurements.

Like the wafer, the grid plate is also fixated by a vacuum chuck which has been machined into the stone (Shown in Figure 5). This is done to obtain a high contact stiffness and to ensure the grid plate lies flat over the entire stone surface. The geometry of this chuck pattern ensures that sufficient vertical stiffness of the scanhead on the grid plate is maintained in all possible locations of the scanhead. 


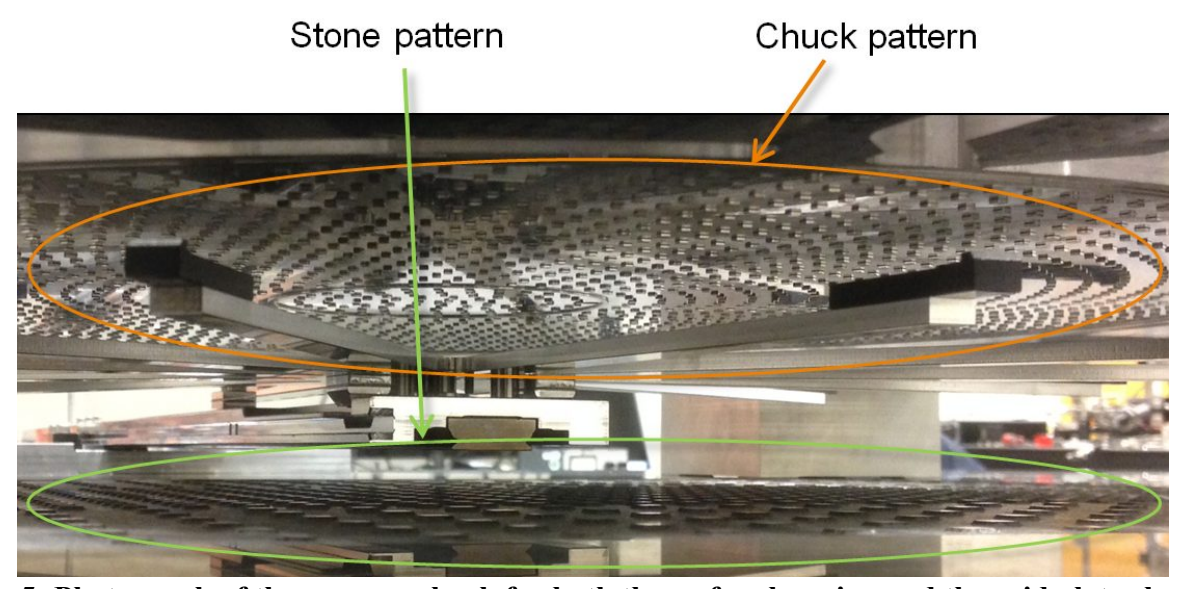

Figure 5: Photograph of the vacuum chuck for both the wafer clamping and the grid plate clamping.

Due to the different materials used, temperature effects on the performance had to be evaluated, as thermal settling of components can result in sudden movements or jerks in the system. Analysis showed that a temperature variance of $1 \mathrm{~K}$ and a maximum slope of $0.1 \mathrm{~K} / \mathrm{hr}$ are practical values for use of this system in a lab environment.

\section{WAFER STAGE}

The wafer needs to make a 2D scanning motion over the AFM tip. All out of plane errors in this movement highly impact the measurement accuracy. Therefore a high vertical stiffness $(>1 \mathrm{e} 8 \mathrm{~N} / \mathrm{m})$ of the wafer chuck is needed w.r.t. the scanhead combined with a low coupling of the $\mathrm{x}, \mathrm{y}$ scanning motion to the vertical $\mathrm{z}$ motion.

On the other hand the stiffness in the scanning directions should be sufficient (not too low to reduce the $\mathrm{x}, \mathrm{y}$ scanning bandwidth) for proper actuation of the stage. These requirements have been met by making the stage monolithic: the wafer chuck is entirely suspended by and also driven through notched hinges.

The actuation of the chuck is performed by piezo actuators which provide a very stiff motion coupling, ensuring proper following of the intended scanning motion profile. Since the stroke of the stage is relatively small with $+/-25 \mu \mathrm{m}$ in both directions, the stresses in the hinges are low enough to ensure sustained operation well within the fatigue limits of the used material.

In order to keep a statically determined layout, an anti-rotation assembly is mounted on top of the wafer stage which only prohibits rotation of the chuck around the vertical axis.

The mass of the chuck including the anti-rotation assembly was reduced to $14 \mathrm{~kg}$ to keep the lowest Eigen frequency high enough to allow for fast scanning and still have enough headroom to use non-sinusoidal motion profiles for scanning. Figure 6 shows the design and realized version of the wafer stage and parallel arms.

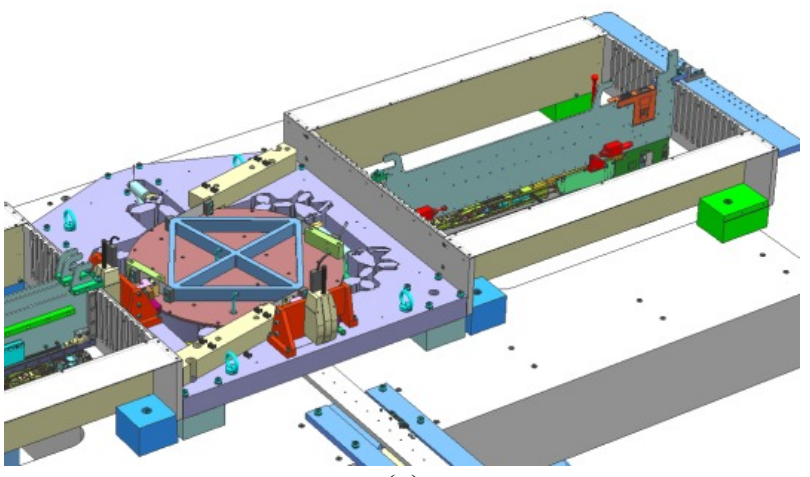

(a)

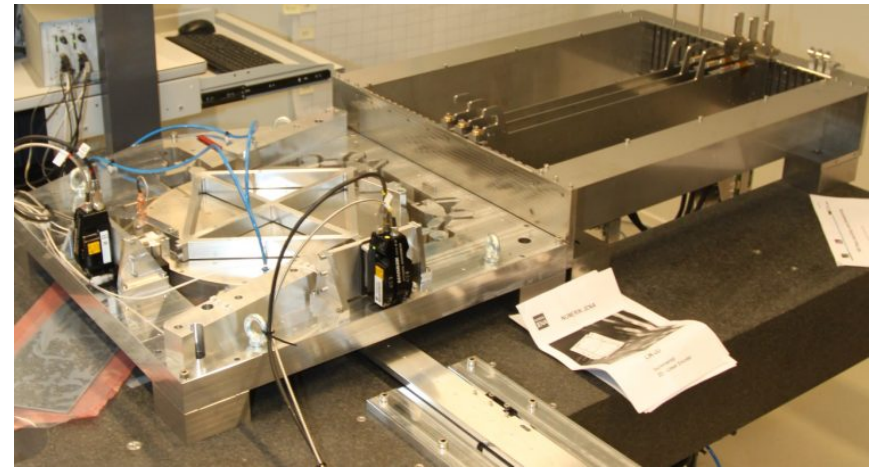

(b)

Figure 6: (a) the CAD of parallel SPM, including the wafer stage in the middle. (b) the realized part of parallel SPM, with wafer stage in the middle. 


\section{POSITIONING UNIT}

The positioning unit (PU) is designed as a cassette which can be easily removed from a cassette box in order to perform maintenance operations. Each PU/cassette incorporates a scanhead, an arm, a linear actuator to extent and retract the arm with its scanhead and the PCB interfaces for the electrical connections towards the control unit.

The cassette box has room for 22 positioning units. Each cassette is inserted into slots on both ends of the cassette box and receives a slight sideways preload when the cassette is inserted fully, eliminating any play. The position along the arm length is repeatable due to a ball in $\mathrm{V}$-groove locating feature.

In our demonstrator two cassette boxes are present, one on each side of the wafer stage. One box will be filled with three positioning units and the second box will have one. This is a minimum number of parallel units which is needed to show the proof of parallelization.

The positioning units can be placed $20 \mathrm{~mm}$ apart, with a $1 \mathrm{~mm}$ gap in between. This restricts the width of each unit to a maximum of $19 \mathrm{~mm}$. In order to fit all the wiring needed to operate and control the unit into this envelope, flex rigid cables have been designed and incorporated into the system. Since the flex-cables also connect to the scanhead, special care has been taken to ensure the stiffness of the bridge between scanhead and arm is sufficiently low, maintaining a high placement accuracy and decoupling of the arm dynamics from the scanhead.

Figure 7 shows the realized version of positioning unit, the mini scanhead and how they are placed in the cassette box.

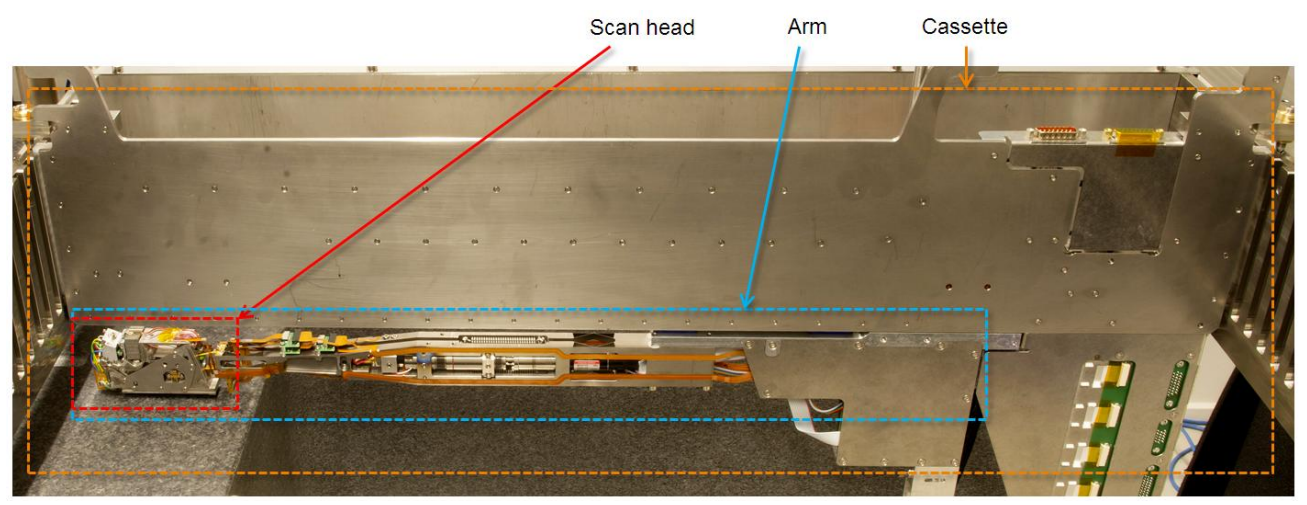

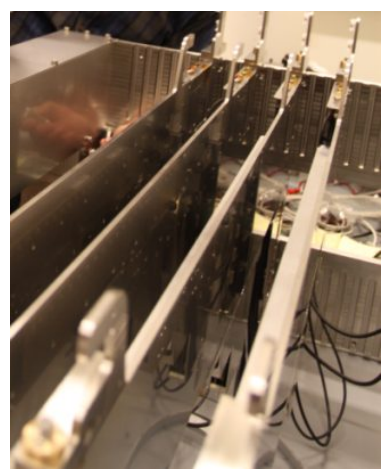

(b)

Figure 7: Photograph of (a) a realized positioning unit, arm and scanhead in a cassette. (b) parallel cassettes in the cassette box.

At the rear of the arm, a custom PCB is placed which forms the interface for the connections coming from the scanhead and arm and also for the larger flex cables going from the arm to the fixed world. These larger flex cables have some slack, facilitating the linear movement of the positioning unit. The fixed world in this case is the back side of the cassette box where regular connectors are used to make removal of a cassette possible.

This second version of the positioning unit has several key improvements over the former version. Figure 8 shows the photography of the realized positioning unit from last year and also the improved version which is suitable for parallelization this year. 


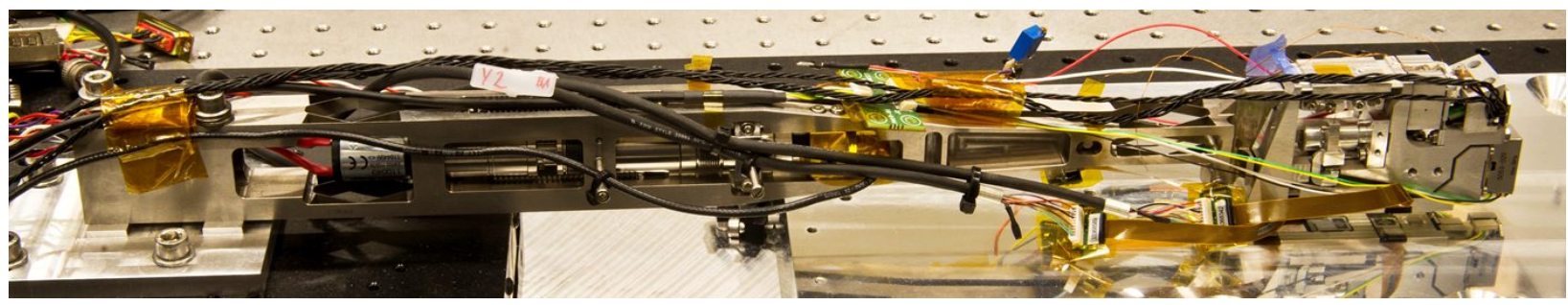

(a)

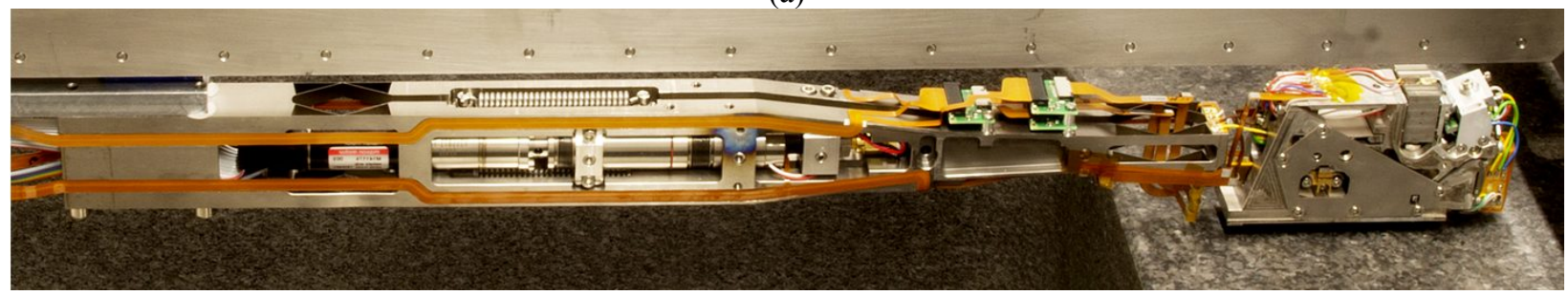

(b)

Figure 8: Photograph of (a) the first version and (b) the second version of positioning unit and scan head.

\section{SCANHEAD}

The positioning unit moves the scanhead in the horizontal plane to the point of interest underneath the wafer. The scanhead has a single mechanism that performs both the landing of its base onto the grid plate as well as the approach of the cantilever tip towards the surface of the wafer. This actuation also ensures that the scanhead is disconnected mechanically from the arm, with exception of the flex rigid cables which have been designed for lowest coupling forces.

One major improvement of the scanhead is the revision of the alignment of the optical column which is part of the optical beam deflection (OBD) detection. To speed up re-alignment of the OBD after tip exchange, the required degrees of freedom were separated into individual adjustments with good orthogonality:

By sliding the barrel in and out (Tz) the focus of the beam on the cantilever can be adjusted. This is done with a small alignment stick which also introduces a welcome reduction ratio for the translation sensitivity.

Tilting the barrel $(\mathrm{Rx})$ moves the focus spot along the cantilever beam and is made possible by spark erosion machining of the scanhead frame, creating elastic elements. The folding mirror used in the OBD previously was not adjustable [5, 6]. In the modified design the mirror is placed in a spark eroded bracket that can elastically be adjusted along one rotational axis (Ry) from outside scanhead. Adjustment of this bracket moves the focus spot perpendicular to the cantilever beam.

The adjustment resolution for Rx and Ry have been improved by using the stiffness of the spark eroded features to obtain a reduction ratio for the stroke. Also a normal hex driver can be used for adjustment.

All three adjustments can be made separately and from outside scanhead without the need to remove parts.

Other improvements that have been incorporated into the scanhead design:

- The kinematic mount of the scanhead on the arm has been modified, becoming much more robust and compact due to the elimination of a fragile strut and leaf spring that were used previously.

- Together with the improved kinematic mount, contact switches have been added to monitor proper landing of the scanhead onto the grid plate as well as proper picking-up by the arm.

- Cover plates were added to the scanhead to improve the stiffness of the scanhead assembly and to provide support for the flex rigid cables.

- In the barrel the xyz positions of the laser diode w.r.t. the lens have the most sensitive tolerances when it comes to the alignment range needed to get the spot onto the cantilever when a complete scanhead is assembled. With a few modifications in the way the diode is mounted in the barrel, the required alignment range is greatly reduced. Being the most critical component, an additional advantage is that the barrel assembly can be assembled and measured separately.

Figure 9 shows the final design and the realized version of the scanhead. 


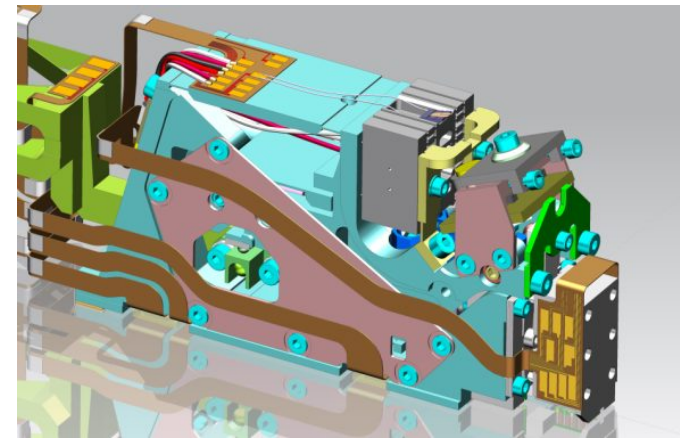

(a)

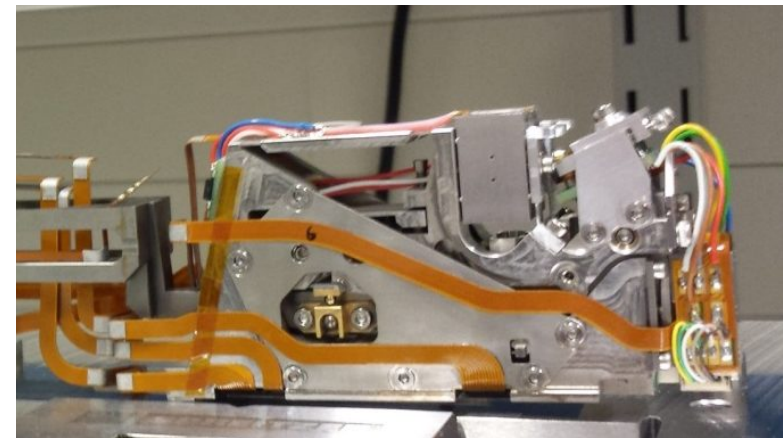

(b)

Figure 9: (a) CAD of the miniature scanhead with flex-cables and (b) the realized version of the scanhead.

\section{WAFER HANDLER}

For the proof of principle of parallelization and high throughput SPM a wafer handling system is not needed, since it is a very common practice with TRL of 9 . However insertion and removal of a wafer into the wafer stage by hand was no option: the $47 \mathrm{~mm}$ high letterbox-sized opening, the long reach needed towards the chuck and the risk of damaging delicate parts in close proximity proved the need for a dedicated wafer handler. Figure 10 illustrates a schematic of the wafer handling system.

Since the lab setup's primary goal is to prove that parallel scanning of a wafer is possible, it is sufficient to reliably attach a wafer with $+/-1 \mathrm{~mm}$ accuracy on the chuck. The handler will be operated manually.

The wafer gripper is made in the form of a spider with slim elastic arms to accommodate a small deviation in the combined flatness of the arm tips where the wafer lies upon. The spider is attached on a linear guide which can manually be moved to insert and retract the wafer to and from the chuck center. When the spider has reached the chuck center, the position of the linear guide unlocks a rotational degree of freedom and also its adjustment so that by tightening a screw the spider can move towards the chuck. This way the handler can only lift the wafer when the wafer is at the chuck center. This eliminates the risk of the wafer crashing into the side of the wafer stage or into the chuck pattern when the linear guide is moved from its park position outside the wafer handler.
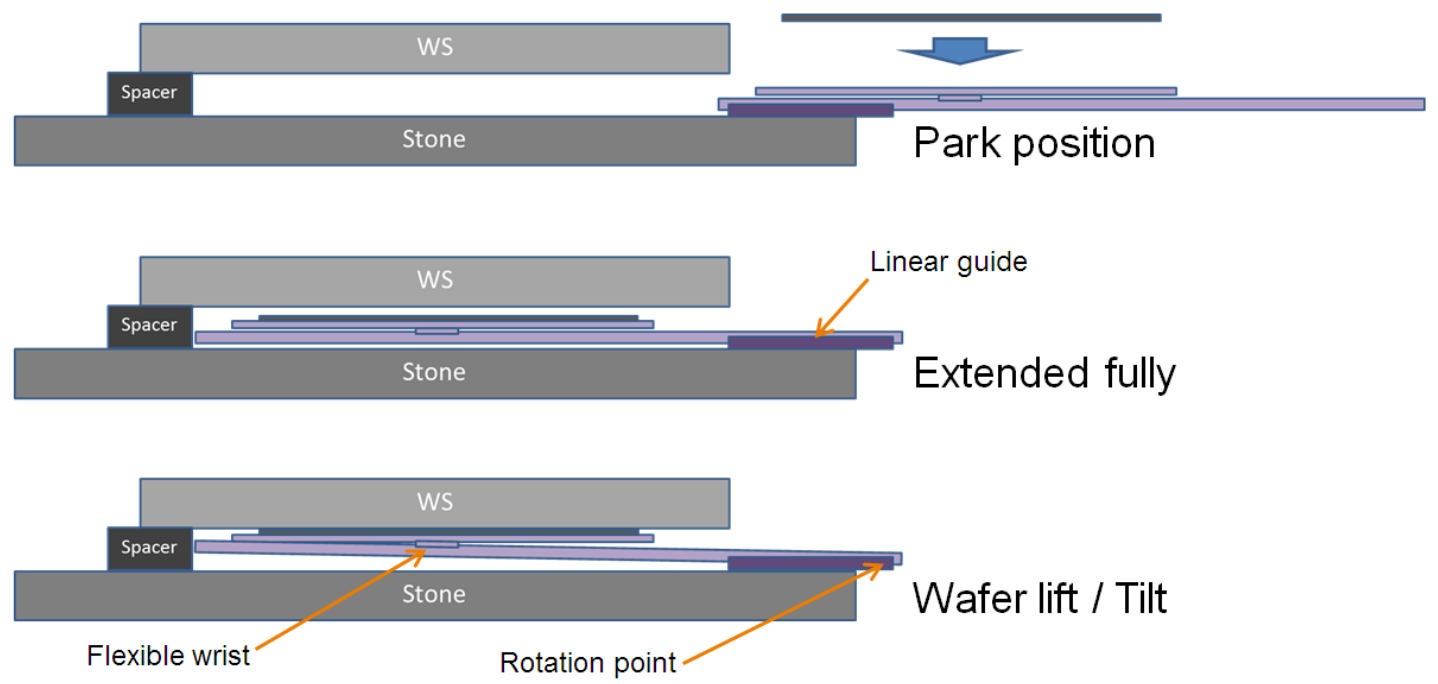

Figure 10: Schematic illustration of manual wafer handling system.

Because the rotation point of the arm lies well outside of the wafer hander, the spider/wafer will near the chuck be at an angle of a few degrees. Underneath the spider there is a flexible wrist with low stiffness so the wafer can adjust itself to the angle of the chuck along two axes. When the wafer is fully seated against the chuck, a compliant spring relief ensures 
that the force on the wafer and the chuck increases only slightly when the wafer handler is lifted even further. An end stop in the arm prevents a hard stop of the arm against the chuck before the entire spring range is used.

In parked position the handler sticks out a long distance, limiting working space and posing a risk for damage to both the handler and people working with it. That is why it has been designed to be easily removable from the stone.
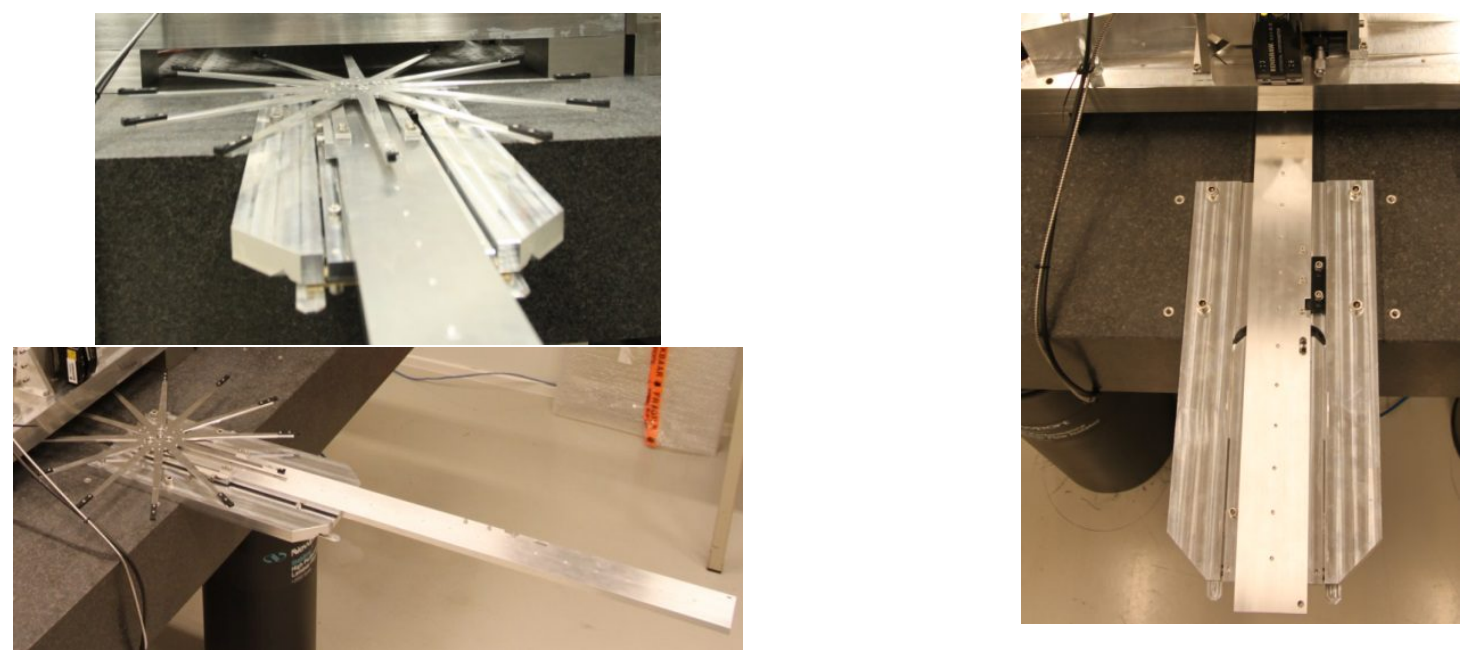

Figure 11: Realized wafer handler.

\section{RESULTS AND DISCUSSION}

A key requirement of the parallel SPM system is that the SPMs and positioning units can be reproducibly manufactured. Especially for parts that require precise assembly steps and are directly connected to the performance of the system this is an area requiring scrutiny. This is the case for the four z-actuators, which performance is sensitive to manufacturing tolerances, alignment of the piezo actuators, and balancing.

In Figure 12 the frequency response of the four z-stages after insertion of the piezo actuators is shown. As can be seen, up to the first resonance frequency, the frequency response is quite flat. Also, the spread of resonance frequency is below $5 \%$. The matching between the z-stages is also good for the displacement gain: when the stroke of the piezo is measured as function of input voltage, the spread is below 5\%. This is shown in Figure 13. Since the cantilever holder and dither piezo are positioned on top of the z-stage, a counterbalance is required at the opposite side. This reduces the resonance frequency a few $\mathrm{kHz}$, so that after balancing the spread in resonance frequency is from 46 to $48 \mathrm{kHz}$. 

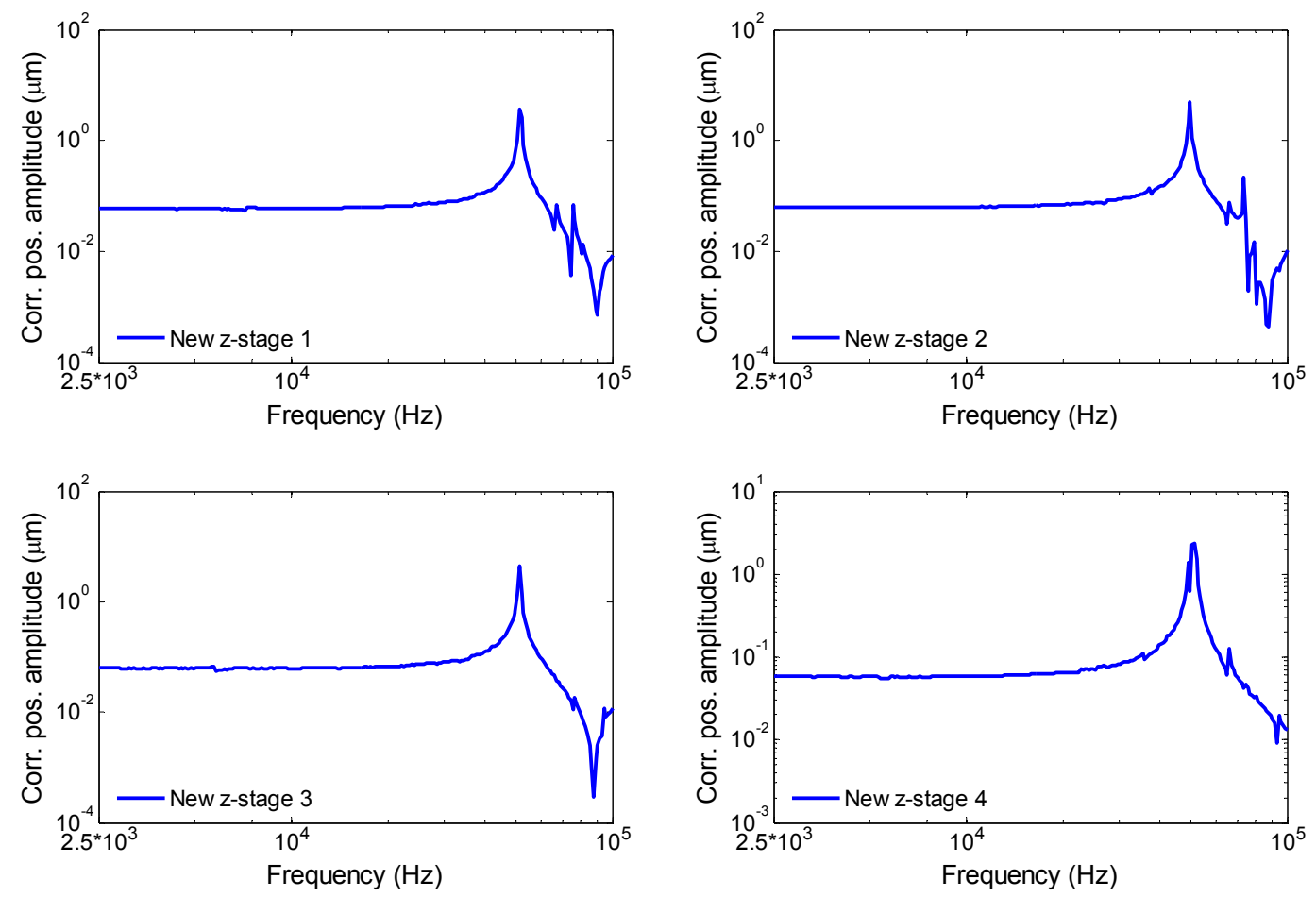

Figure 12. Frequency response of the four $z$-stages after insertion of the piezo actuators. All 4 resonance frequencies lie between 49.5 and $51.5 \mathrm{kHz}$, indicating a well-controlled fabrication of the z-stages.

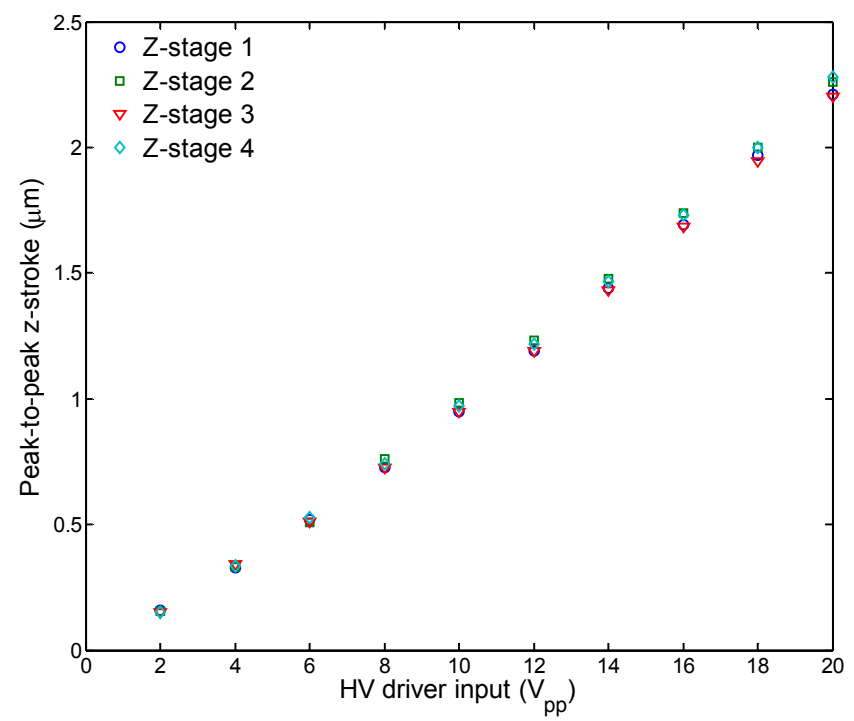

Figure 13. Peak-to-peak stroke of the z-guidance at low frequency $(200 \mathrm{~Hz})$. Displacement as function of driving voltage exhibits a spread $<5 \%$.

The integration of the electrical connections into flex-rigid PCBs was tested as well. First of all, functional tests showed all signal lines were properly connected. Secondly we tested whether the longer signal lines had a negative influence on 
the OBD. In Figure 14 we show the OBD performance as measured through the flex-rigid PCBs. As can be seen, there is a clean resonance peak when an Olympus AC55TS cantilever is excited using the dither piezo (i.e. no strange small peaks or jags near the main resonance peak). Secondly, the OBD noise level is low enough to observe the thermal noise of this high-speed cantilever. This is crucial for making fast high-quality scans.

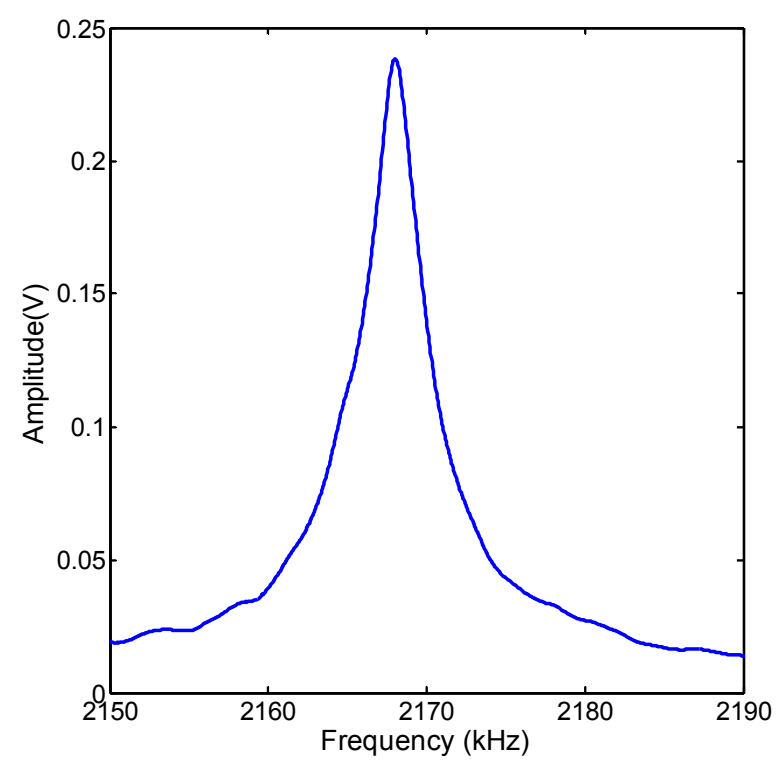

(a)

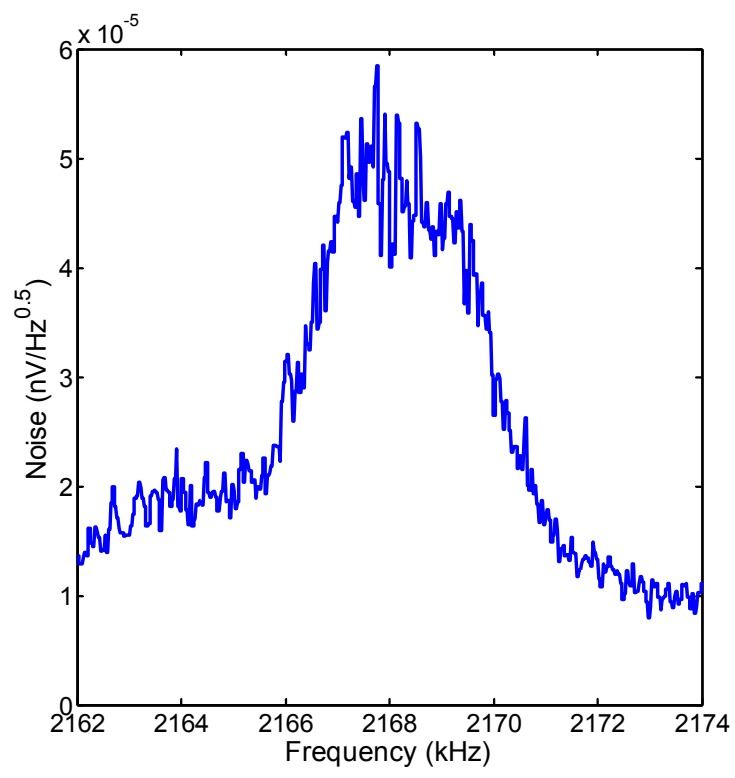

(b)

Figure 14. Frequency response (a) and thermal noise (b) of a $2 \mathrm{MHz}$ Olympus AC55TS probe measured with the full cassette, arm, and scanhead combination.

Another check that was performed to test whether the flex-rigid exhibits significant adverse effects was a cross-talk measurement. Since in the flex-rigid PCBs the signal lines for the high voltage piezo actuators are near the OBD measurement signal lines, we investigated whether the actuation of the z-stage had a significant effect on the free-air amplitude of the cantilever. To this end we measured the free-air amplitude while a $14.3 \mathrm{~V}, 7 \mathrm{kHz}$ test signal was applied to the Z-actuator input. To distinguish between mechanical cross-talk that is unavoidable and electrical cross-talk which will come in addition to that we also applied the same signal while the z-actuator was bypassed with a short at the end of the flex-rigid pcb's. This way, there is no mechanical actuation, but the currents associated with actuation will flow through the cables and flex-rigid pcb's and potentially slightly alter the OBD signal levels by inductive coupling. The free air amplitude was set to $214 \mathrm{mV}$.

The result of this is shown in Figure 15. In this, the mechanical cross-talk is clearly visible. Nonetheless, the $7 \mathrm{kHz}$ variation in amplitude is a factor $2.7^{*} 10^{3}$ times lower than the mean amplitude, despite the relatively large mechanical excitation ( $14.3 \mathrm{~V}$ is about $10 \%$ of the full actuation range). If the actuator is then shorted, the peak greatly diminishes, indicating that mechanical cross-talk dominates the electrical cross-talk and the flex-rigids are not reducing system performance through this mode. 


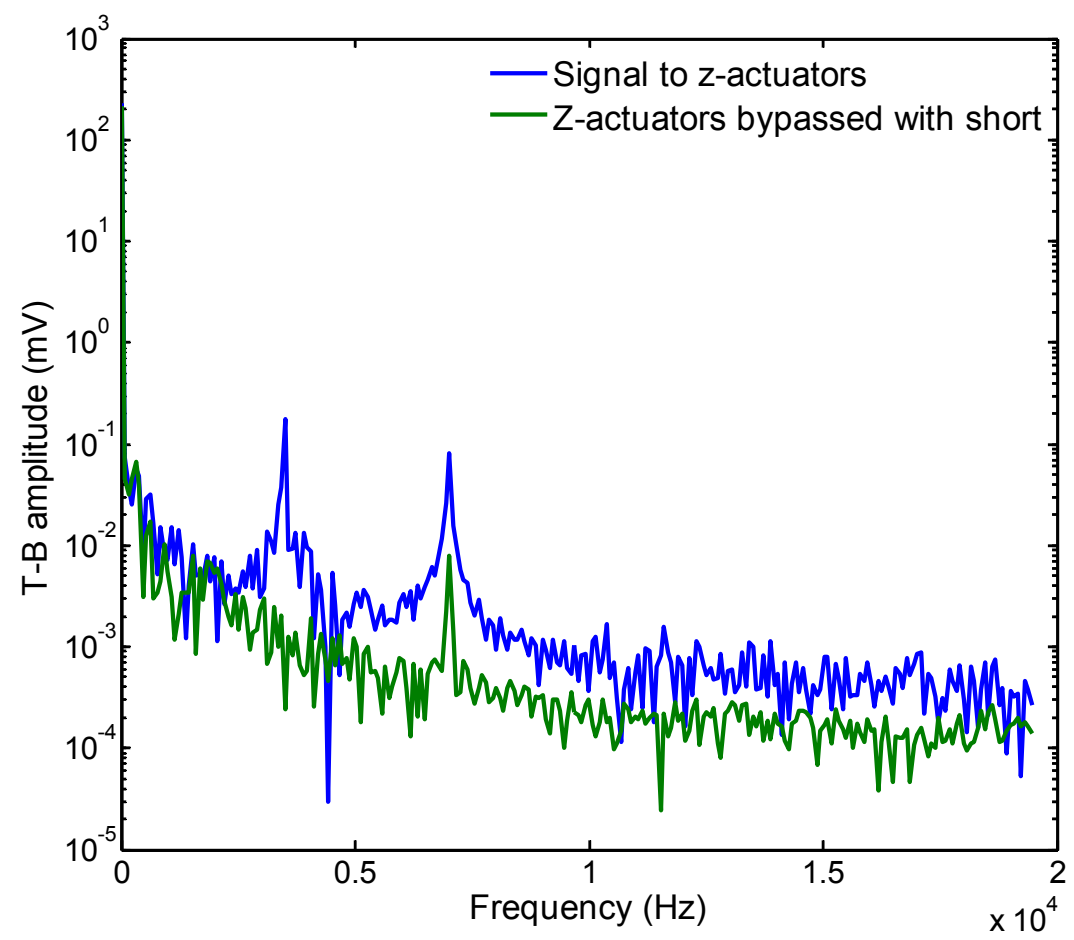

Figure 15. Frequency spectrum of the demodulated OBD signal showing modulation of the free-air amplitude due to a $7 \mathrm{kHz}$, 14.3V signal applied at the $z$-actuator input of the arm+scanhead with flex-rigid pcb. When the z-piezo's are shorted at the scanhead, modulation of the free-air amplitude greatly decreases.

In Figure 16 two scans of a calibration line sample are shown, one without flex-rigid pcb (i.e. OBD and piezo-actuators connected using regular cables) and using an Arrow UHF cantilever, and one with the full arm+scanhead including the flex-rigid pcb's using an Olympus AC55TS probe. Although there is a small difference in ultimate image quality due to the use of different cantilevers, overall there are no indications that the new flex-rigid pcb's negatively influence the picture quality. 


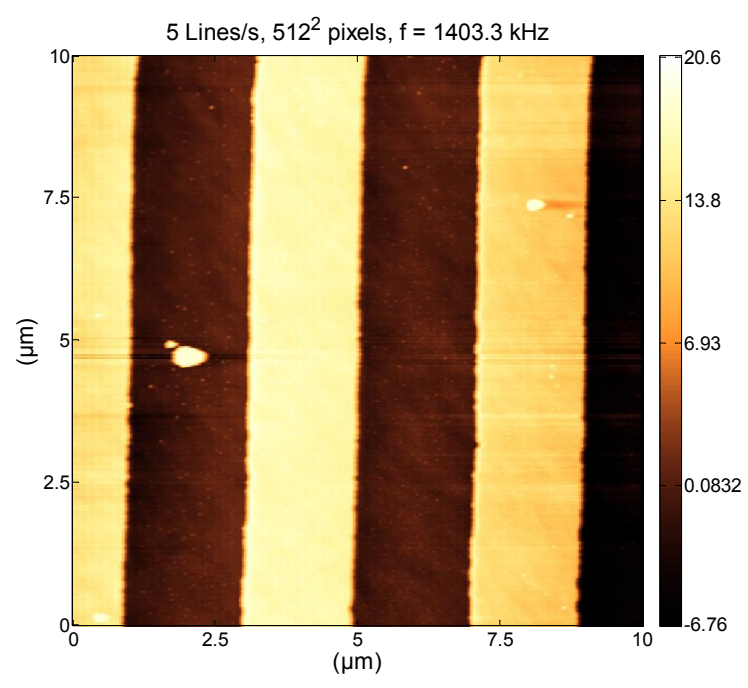

(a)

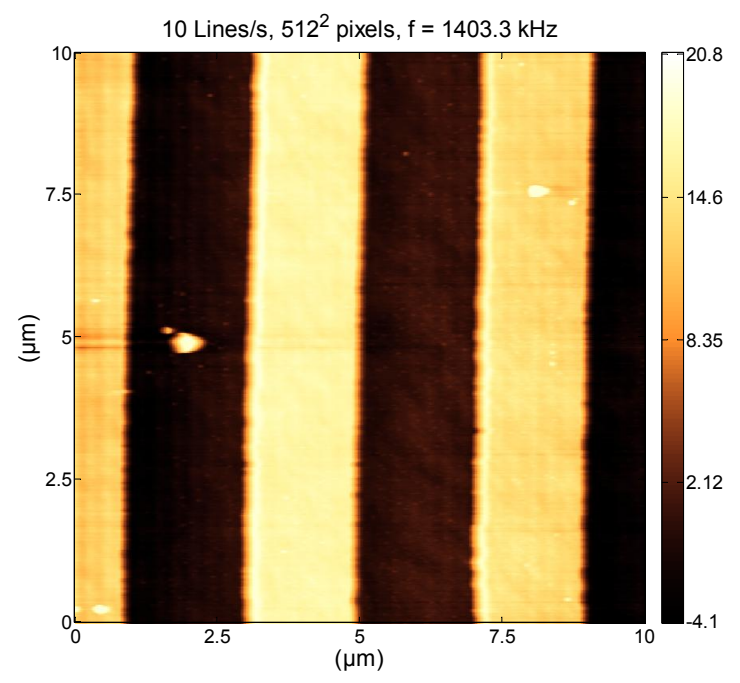

(c)

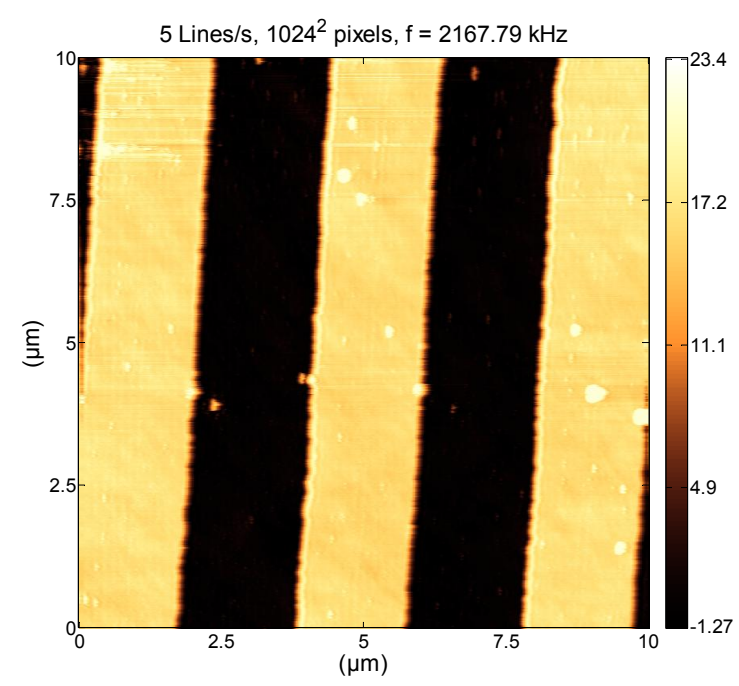

(b)

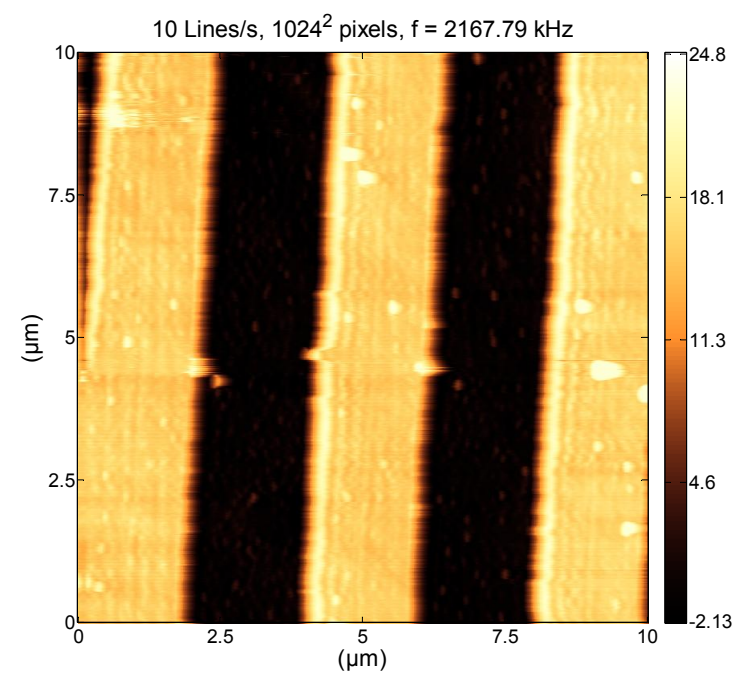

(d)

Figure 16. Measurements of a calibration line sample at $10 \mathrm{~Hz}$. Sample had a step height of $23 \mathrm{~nm}$. (a) Measured without flexrigid pcb's and an Arrow UHF cantilever, 5 lines/s. (b) Measured with full arm+scanhead including flex-rigid pcb using an Olympus AC55TS probe, 5 lines/s. (c) Measured without flex-rigid pcb's and an Arrow UHF cantilever, 10 lines/s. (d) Measured with full arm+scanhead including flex-rigid pcb using an Olympus AC55TS probe, 10 lines/s. 


\section{CONCLUSION AND FUTURE WORK}

In conclusion, we have shown the proof of principle of parallel AFM equipment in terms of sub-systems, for metrology and inspection. With 4 parallel AFMs system we show the feasibility of all technical challenges involved. The next step is to fully integrate the system and run tests on full wafer against specific application requirements. The system can then be scaled up for the ultimate required throughput by parallelizing more than 40 scanheads.

*hamed.sadeghianmarnani@tno.nl

\section{ACKNOWLEDGMENT}

The research described in this paper was part of the E450EDL project, for which we gratefully acknowledge funding by the ECSEL Joint Undertaking and the Netherlands Enterprise Agency (RVO).

\section{References}

1. Jason Osborne, Shuiqing Hu, Haiming Wang, Yan Hu, , Jian Shi, Sean Hand, Chanmin Su, "High-speed atomic force microscopy for patterned defect review" in Proc. SPIE 8681, Metrology, Inspection, and Process Control for Microlithography XXVII, 86813C, San Jose, 2013

2. Yueming Hua, Cynthia Coggins, Yong-ha Lee, Jung-min Lee, Kyung-deuk Ryang, Sang-il Park. "New 3Dimensional AFM for CD Measurement and Sidewall Characterization", Proceedings of SPIE (2011), Vol. 7971, 797118.

3. H. Sadeghian ; N. B. Koster and T. C. van den Dool "Introduction of a high throughput SPM for defect inspection and process control", Proc. SPIE 8681, Metrology, Inspection, and Process Control for Microlithography XXVII, 868127 (2013).

4. H. Sadeghian; T. C. van den Dool; W. E. Crowcombe; R. W. Herfst; J. Winters, et al. "Parallel, miniaturized scanning probe microscope for defect inspection and review", Proc. SPIE 9050, Metrology, Inspection, and Process Control for Microlithography XXVIII, 90501B (2014).

5. R. J. F. Bijster, J. de Vreugd, H. Sadeghian, Phase lag deduced information in photo-thermal actuation for nano-mechanical systems characterization, Applied Physics Letters, 105(7), 073109 (2014).

6. R.W. Herfst, W.A. Klop, M. Eschen, T.C. van den Dool, N.B. Koster, H. Sadeghian, Systematic characterization of optical beam deflection measurement system for micro and nanomechanical systems, Journal of Measurement 56, 104-116 (2014).

7. H. Sadeghian, RW Herfst, TC van den Dool, WE Crowcombe, J Winters, High-throughput Parallel SPM for Metrology, Defect, and Mask Inspection, Proc. SPIE 9231, 30th European Mask and Lithography Conference, 92310B (2014). 